\title{
Frecuencia de reactividad cutánea hacia alérgenos alimentarios en pacientes alérgicos
}

Heriberto Camero-Martínez, ${ }^{1}$ Aída Inés López-García, ${ }^{1}$ Daniela Rivero-Yeverino, ${ }^{1}$ Chrystopherson Gengyny Caballero-López, ${ }^{1}$ Oswaldo Arana-Muñoz, ${ }^{1}$ Sergio Papaqui-Tapia, ${ }^{1}$ Isabel Cristina Rojas-Méndez, ${ }^{1}$ Elizabeth Vázquez-Rojas ${ }^{1}$

\begin{abstract}
Background: Food allergy is deemed to have a worldwide prevalence ranging from 2 to $10 \%$. Objective: To determine the frequency of skin reactivity to food allergens by age groups. Methods: Cross-sectional, descriptive, prolective, observational study. Patients aged from 2 to 64 years with symptoms consistent with allergic disease were included. Skin prick tests were carried out with food allergens. Frequencies and percentages were estimated.

Results: One-hundred and ninety-one patients were included, out of which $63.4 \%$ were females. Mean age was 22.5 years; $19.3 \%$ showed positive skin reactivity to at least one food. Distribution by age group was as follows: preschool children $13.5 \%$, schoolchildren $24.3 \%$, adolescents $2.7 \%$ and adults $59.5 \%$. Diagnoses included allergic rhinitis in $84.3 \%$, asthma in $19.4 \%$, urticaria in $14.1 \%$ and atopic dermatitis in $8.4 \%$. Positive skin reactivity frequency distribution in descending order was: soybeans with $5.2 \%$, peach with $4.7 \%$, grapes, orange and apple with $3.6 \%$, nuts with $3.1 \%$, pineapple, avocado, tomato and tuna with $2.6 \%$.

Conclusion: The frequency of skin reactivity to food allergens was similar to that reported in the national and Latin American literature, but sensitization to each specific allergen varied for each age group.
\end{abstract}

Keywords: Food allergy; Skin prick tests

Este artículo debe citarse como: Camero-Martínez H, López-García Al, Rivero-Yeverino D, Caballero-López CG, Arana-Muñoz, Papaqui-Tapia S, Rojas-Méndez IC, Elizabeth Vázquez-Rojas E. Frecuencia de reactividad cutánea hacia alérgenos alimentarios en pacientes alérgicos. Rev Alerg Mex. 2017;64(3):291-297

${ }^{1}$ Hospital Universitario de Puebla, Servicio de Alergia e Inmunología Clínica. Puebla, Puebla, México
Correspondencia: Heriberto Camero-Martínez. drcamero@hotmail.com

Recibido: 2017-05-15

Aceptado: 2017-06-14 


\section{Resumen}

Antecedentes: Se considera que la alergia alimentaria tiene una prevalencia mundial de 2 a $10 \%$. Objetivo: Determinar la frecuencia de reactividad cutánea hacia alérgenos alimentarios por grupos de edad.

Métodos: Estudio observacional, descriptivo, transversal y prolectivo. Se incluyeron pacientes de 2 a 64 años de edad con cuadro compatible de enfermedad alérgica. Se efectuaron pruebas por punción cutánea con alérgenos alimentarios. Se estimaron frecuencias y porcentajes.

Resultados: Se incluyeron 191 pacientes, $63.4 \%$ fue del sexo femenino. La edad promedio fue 22.5 años; 19.3 \% mostró reactividad cutánea positiva al menos a un alimento. La distribución por grupo etario fue la siguiente: preescolares $13.5 \%$, escolares $24.3 \%$, adolescentes $2.7 \%$ y adultos $59.5 \%$. Los diagnósticos fueron rinitis alérgica $84.3 \%$, asma $19.4 \%$, urticaria $14.1 \%$ y dermatitis atópica $8.4 \%$. La distribución de la frecuencia de reactividad cutánea positiva en orden descendente fue $5.2 \%$ a soya, $4.7 \%$ a durazno, $3.6 \%$ a uva, naranja y manzana, $3.1 \%$ a nuez y $2.6 \%$ a piña, aguacate, tomate y atún.

Conclusión: La frecuencia de reactividad cutánea para alérgenos alimentarios fue similar a la informada en la literatura nacional y latinoamericana, pero la sensibilización para cada alérgeno específico varió en cada grupo etario.

Palabras clave: Alergia alimentaria; Pruebas cutáneas por punción

\section{Abreviaturas y siglas}

DBPCFC, reto doble ciego controlado con placebo

\section{Introducción}

La alergia alimentaria se define como un efecto adverso a la salud provocado por una respuesta inmunológica específica que ocurre de forma reproducible con la exposición a un alimento determinado. ${ }^{1}$

En las últimas 2 décadas, la alergia alimentaria se ha convertido en un importante problema de salud pública que afecta a personas de todas las edades $\mathrm{y}$ en todas las regiones. Se considera que tiene una prevalencia mundial de 2 a $10 \%$, de 4 a $5 \%$ en la población infantil y de 2 a $3 \%$ en adultos. Se estima que aproximadamente 11 a 26 millones de la población europea sufre alergia a alimentos; en Asia se informa una prevalencia entre 1.2 y $6.5 \%$; en Estados Unidos afecta a $6 \%$ de los niños menores de 5 años y de 3.5 a $4 \%$ de la población adulta; en ese país, la alergia alimentaria es responsable de 30000 episodios de anafilaxia y 100 muertes por año. En México se reporta una prevalencia de $1 \%$ en los niños. . $^{2,3,4,5,6}$

Los alérgenos alimentarios son generalmente glucoproteínas con peso molecular que oscila entre 10 y $70 \mathrm{kDa}$; de estos, los más comunes en los lac- tantes y los niños son la leche de vaca, huevo de gallina, cacahuate, frutos secos, soya y trigo, mientras que en los adultos son cacahuate, nueces, mariscos, frutas y verduras. ${ }^{7}$

De acuerdo con la distribución geográfica, en países europeos los alimentos más frecuentes que producen alergia son la leche de vaca, huevo, trigo, soya, cacahuate, frutos secos, pescado y mariscos. ${ }^{8}$ En Estados Unidos existe una prevalencia similar para dichos alimentos. ${ }^{9}$ En México, los alimentos más frecuentemente identificados mediante las pruebas cutáneas fueron queso, cacahuate, atún, frijol, chile y camarón; los resultados variaron según los diferentes grupos de edad. ${ }^{10}$

Conforme a su inmunopatología, la alergia alimentaria se divide en 3 categorías: reacciones mediadas por IgE, reacciones mediadas por células y reacciones mixtas, mediadas por IgE y células. ${ }^{1,4}$ Las reacciones no mediadas por IgE incluyen las tipos II, III y IV según Gell y Coombs; respecto a las 2 primeras aún existe poca evidencia que desempeñen un papel significativo en los trastornos alérgicos a alimentos. 
La hipersensibilidad tipo IV, o mediada por células, ha sido implicada en los trastornos en los que la aparición de los síntomas se produce varias horas después de la ingestión del alérgeno alimentario responsable. ${ }^{1,7}$

Los síntomas clínicos de alergia a los alimentos se presentan en una variedad de síndromes. Las manifestaciones cutáneas incluyen principalmente urticaria, angioedema y dermatitis atópica; las gastrointestinales, prurito de boca y labios, dolor abdominal, vómito, diarrea y estreñimiento; las respiratorias, rinorrea, prurito y constipación nasal, tos, sibilancias y disnea. Pueden aparecer entidades clínicas como reflujo gastroesofági$\mathrm{co}$, esofagitis eosinofilica alérgica, gastroenteritis eosinofílica alérgica, síndrome de enterocolitis inducida por proteínas, proctitis alérgica, síndrome de Heiner, asma y rinitis crónica. La anafilaxia es la manifestación generalizada más severa. ${ }^{1,4,7,11}$

El diagnóstico de alergia a los alimentos se basa en una historia clínica completa, en la que se incluye el diario de alimentos. Entre los métodos diagnósticos se encuentran las pruebas cutáneas por punción, determinación de IgE sérica específica a alimentos por diferentes métodos y la prueba de parche. La positividad de estos indica sensibilización a un alimento específico, sin embargo, no siempre se asocian con manifestaciones clínicas. El reto doble ciego controlado con placebo (DBPCFC) se considera el "estándar de oro" para el diagnóstico, sin embargo, genera riesgo de anafilaxia para el paciente, por lo que es preferible utilizar alternativas más seguras. ${ }^{11,12,13,14,15,16}$

La prueba cutánea es un elemento importante en el diagnóstico de alergia mediada por IgE; entre sus diversas ventajas se encuentra su simplicidad, rapidez para obtener resultados, bajo costo y alta sensibilidad, que por lo general es de 75 a $90 \%$.

Con la finalidad de actualizar o mantener el panel de alérgenos alimentarios para pruebas cutáneas y optimizar la calidad de atención médica del individuo alérgico, se propuso la realización del presente estudio, para determinar la frecuencia de reactividad cutánea para alérgenos alimentarios en los pacientes incidentes del servicio de Alergia e Inmunología Clínica del Hospital Universitario de Puebla, Puebla, México.

\section{Métodos}

Se realizó un estudio observacional, descriptivo, transversal y prolectivo. Se incluyeron pacientes de uno y otro sexos, con edades comprendidas entre 2 y 64 años, con cuadro clínico de enfermedad alérgica, que acudieron por primera vez al Servicio de Alergia e Inmunología Clínica del Hospital Universitario de Puebla en un periodo de 11 meses. Se realizaron pruebas cutáneas por punción con 35 alérgenos alimentarios (Allergomex ${ }^{\circledR}$ ) considerando peso/volumen: uva, piña, naranja, limón, ciruela, papaya, melón, plátano, fresa, manzana, durazno, nuez, aguacate, tomate, papa, zanahoria, chícharo, cacahuate, frijol, soya, maíz, arroz, trigo, carnero, carne de puerco, carne de res, pollo, camarón, atún, clara de huevo, yema de huevo, chocolate y leche. Además, se utilizó histamina como control positivo y solución glicerinada como control negativo.

Para la evaluación se utilizaron los criterios de la Guía mexicana de inmunoterapia. Se estimaron parámetros estadísticos descriptivos: frecuencias, porcentajes y mediciones de dispersión, como desviación estándar.

\section{Resultados}

Se incluyeron 191 pacientes, de los cuales $63.4 \%$ fue del sexo femenino. La edad promedio fue de 22.5 \pm 17.6 años. El $19.3 \%$ mostró reactividad cutánea positiva al menos a un alimento, de esta proporción $59.5 \%$ era adulto, $24.3 \%$ escolar, $13.5 \%$ preescolar y $2.7 \%$ adolescente (Figura 1 ).

Entre los diagnósticos encontramos rinitis alérgica en $84.3 \%$, asma en $19.4 \%$, urticaria en $14.1 \%$ y dermatitis atópica en $8.4 \%$. La distribución de frecuencia de reactividad cutánea positiva en el grupo general de pacientes en orden descendente fue $5.2 \%$ a soya, $4.7 \%$ a durazno, $3.6 \%$ a uva, naranja y manzana; $3.1 \%$ a nuez y $2.6 \%$ a piña, aguacate, tomate y atún; $2 \%$ a leche, chocolate, maíz, papaya, limón, zanahoria y carnero; $1.6 \%$ a clara de huevo, yema de huevo, trigo, melón y papa; $1 \%$ a camarón, arroz y ciruela; $0.6 \%$ a cacahuate, frijol y carne de puerco (Figura 2).

En los preescolares se encontraron como alimentos más frecuentes el durazno y la naranja; en los escolares, uva y aguacate; en los adolescentes, naranja, leche, melón y durazno; en los adultos, soya, manzana y nuez (Cuadro 1).

\section{Discusión}

En la población de estudio, se encontraron manifestaciones respiratorias primordialmente de la vía aérea superior, seguidas de síntomas cutáneos, como se ha informado en la literatura nacional e internacional; los 


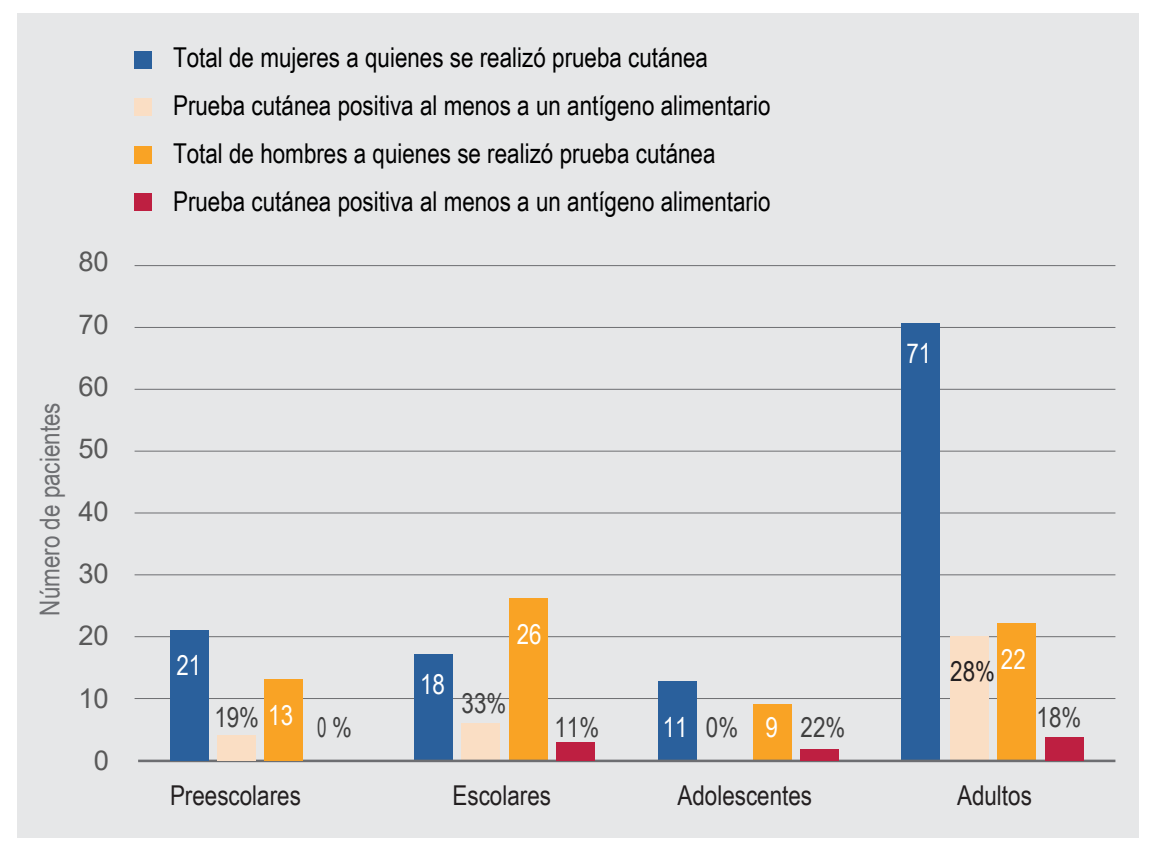

Figura 1. Porcentaje de reactividad cutánea al menos a un alérgeno alimentario por grupo etario y sexo. adultos constituyeron el grupo más afectado.,4,6,19,20,21 En nuestra investigación, las mujeres representaron el mayor porcentaje con prueba cutánea positiva; hasta el momento no hay estudios que comparen la diferencia de reactividad cutánea entre los sexos.

La frecuencia de reactividad cutánea para alérgenos alimentarios es similar a la informada en la literatura nacional y Latinoamérica, sin embargo, hubo variación en la sensibilización a alimentos específicos en los diferentes grupos de edad. ${ }^{10,19,20}$ En los adultos, los alimentos de mayor prevalencia concordaron con los reportados en otras investigaciones., ${ }^{7,9,10,19}$ En cuanto a los niños, destacó la alta prevalencia de reactividad para durazno, aguacate y piña, lo que contrasta con los hallazgos de otros estudios en los que se indica leche de vaca, huevo, cacahuate, y trigo. ${ }^{7,8,22}$

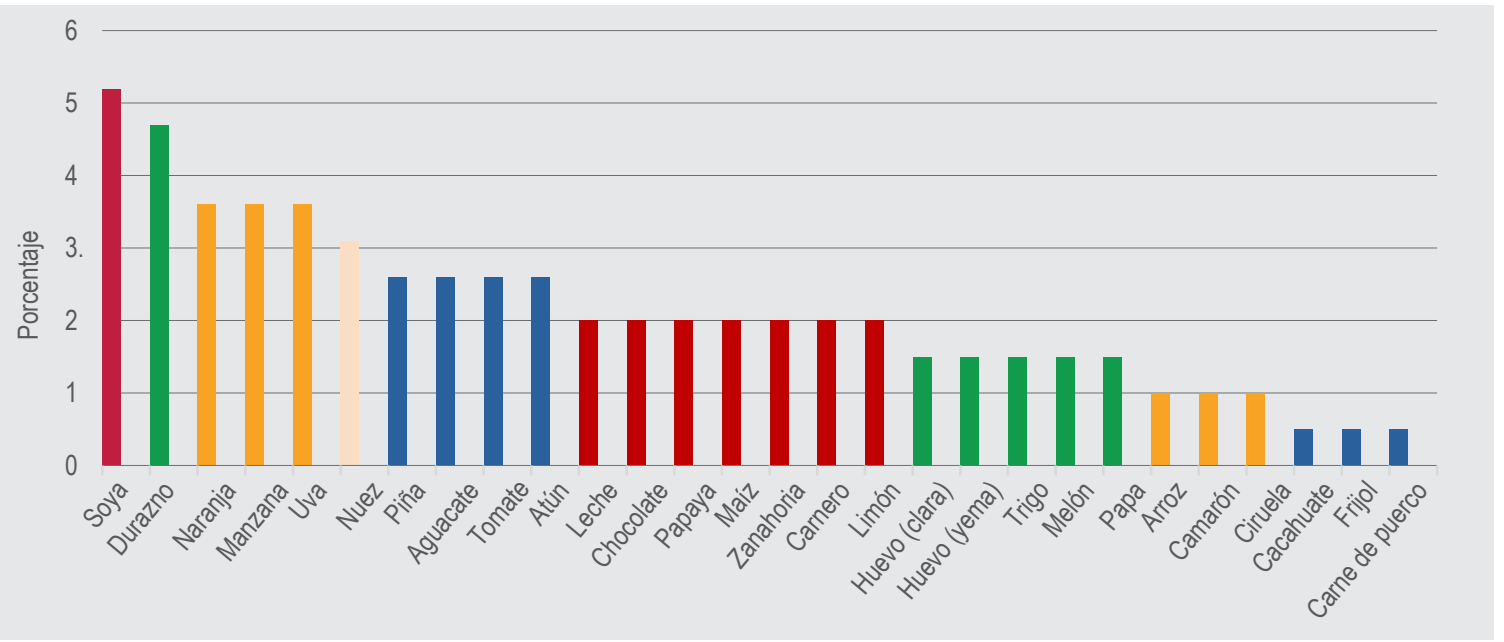

Figura 2. Principales alimentos positivos en las pruebas cutáneas del grupo general de pacientes. 
Camero-Martínez $\mathrm{H}$ et al. Reactividad cutánea hacia alérgenos alimentarios en pacientes alérgicos

Cuadro 1. Frecuencia de reactividad cutánea para alérgenos alimentarios por grupo etario

\begin{tabular}{|c|c|c|c|c|c|}
\hline Grupo etario & Alimento & Frecuencia & Grupo etario & Alimento & Frecuencia \\
\hline \multirow{9}{*}{ Preescolares } & Durazno & 5 & \multirow{4}{*}{ Adolescentes } & Naranja & 2 \\
\hline & Naranja & 3 & & Durazno & 1 \\
\hline & Piña & 1 & & Melón & 1 \\
\hline & Limón & 1 & & Leche & 1 \\
\hline & Melón & 1 & \multirow{27}{*}{ Adultos } & Soya & 9 \\
\hline & Manzana & 1 & & Manzana & 5 \\
\hline & Zanahoria & 1 & & Nuez & 5 \\
\hline & Atún & 1 & & Maíz & 4 \\
\hline & Yema & 1 & & Carnero & 4 \\
\hline \multirow{22}{*}{ Escolares } & Uva & 3 & & Uva & 4 \\
\hline & Aguacate & 3 & & Chocolate & 4 \\
\hline & Durazno & 2 & & Tomate & 3 \\
\hline & Arroz & 2 & & Zanahoria & 3 \\
\hline & Camarón & 2 & & Papa & 3 \\
\hline & Piña & 2 & & Atún & 3 \\
\hline & Papaya & 2 & & Clara & 3 \\
\hline & Limón & 2 & & Piña & 2 \\
\hline & Tomate & 2 & & Aguacate & 2 \\
\hline & Leche & 2 & & Ciruela & 2 \\
\hline & Naranja & 1 & & Papaya & 2 \\
\hline & Melón & 1 & & Trigo & 2 \\
\hline & Fresa & 1 & & Pollo & 2 \\
\hline & Manzana & 1 & & Yema & 2 \\
\hline & Nuez & 1 & & Naranja & 1 \\
\hline & Soya & 1 & & Limón & 1 \\
\hline & Trigo & 1 & & Plátano & 1 \\
\hline & Atún & 1 & & Durazno & 1 \\
\hline & & & & Frijol & 1 \\
\hline & & & & Puerco & 1 \\
\hline & & & & Leche & 1 \\
\hline & & & & Cacahuate & 1 \\
\hline
\end{tabular}


Es probable que las diferencias en los hábitos alimentarios de cada región geográfica influyan en la sensibilización y, por lo tanto, en la frecuencia de la reactividad cutánea, lo cual por otra parte deberá ser evaluado regularmente en función de la optimi- zación del abordaje diagnóstico terapéutico del paciente con cuadro clínico de alergia alimentaria.

De estos hallazgos podría derivarse la pertinencia de incluir a los alérgenos que en el presente análisis presentaron la mayor prevalencia.

\section{Referencias}

1. Nowak-Wegrzyn A, Burks W, Sampson H. Reactions to Foods. En: Adkinson N, Bochner B, Burks A, Busse W, Holgate S, Lemanske R, et al. Middleton's Allergy: Principles and Practice. Volumen II. Octava edición. USA: Mosby; 2014. p. 1310-1335.

2. Burks AW, Jones SM, Boyce JA, Sicherer SH, Wood RA, Assa'ad A, et al. NIAID-sponsored 2010 guidelines for managing food allergy: applications in the pediatric population. Pediatrics. 2011;128(5):955965. DOI: http://dx.doi.org/10.1542/peds.2011-0539

3. Burks AW, Tang M, Sicherer S, Muraro A, Eigenmann PA, Ebisawa M, et al. ICON: food allergy. J Allergy Clin Immunol. 2012;129(4):906-920. DOI: http://dx.doi.org/10.1016/j.jaci.2012.02.001

4. Fiocchi A, Sampson HA, Bahna SL, Lack G. Food Allergy. En: Pawankar R, Canonica GW, Holgate ST, Lockey RF, editores. WAO White Book on Allergy. USA: World Allergy Organization; 2013. p. 47-52.

5. Shek LP, Cabrera-Morales EA, Soh SE, Gerez I, Ng PZ, Yi FC, et al. A population-based questionnaire survey on the prevalence of peanut, tree nut and shellfish allergy in 2 Asian populations. J Allergy Clin Immunol. 2010;126(2):324-331. DOI: http://dx.doi.org/10.1016/j.jaci.2010.06.003

6. Rodríguez-Ortiz P, Muñoz-Mendoza D, Arias-Cruz A, González-Díaz SN, Herrera-Castro D, VidaurriOjeda AC. Características epidemiológicas de pacientes con alergia a alimentos atendidos en el Centro Regional de Alergias e Inmunología Clínica de Monterrey. Rev Alerg Mex. 2009;56(6):185-191.

7. Chapman JA, Bernstein IL, Lee RE, Oppenheimer J, Nicklas RA, Portnoy JM, et al. Food allergy: A practice parameter. Ann Allergy Asthma Immunol. 2006;96(3 Suppl 2):1-68.

8. Nwaru BI, Hickstein L, Panesar SS, Roberts G, Muraro A, Sheikh A, et al. Prevalence of common food allergies in Europe: a systematic review and meta-analysis. Allergy. 2014;69(8):992-1007. DOI: http:// dx.doi.org/10.1111/all.12423

9. Sicherer SH. Epidemiology of food allergy. J Allergy Clin Immunol. 2011;127(3):594-602. DOI: http:// dx.doi.org/10.1016/j.jaci.2010.11.044

10. González-Díaz S, Arias-Cruz A, Domínguez-Sansores LA, Galindo-Rodríguez G, Leal-Villareal L, Canseco-Villareal JI, et al. Sensibilización a alérgenos alimentarios en pacientes del Servicio de Alergia del Hospital Universitario de la UANL, Monterrey. Med Univer. 2013;15(59):59-63.

11. Sicherer SH, Sampson HA. Food allergy: Epidemiology, pathogenesis, diagnosis and treatment. J Allergy Clin Immunol. 2014;133(2):291-307;quiz 308. DOI: http://dx.doi.org/10.1016/j.jaci.2013.11.020

12. Berin MC. Mechanisms of allergic sensitization to foods: bypassing immune tolerance pathways. Immunol Allergy Clin North Am. 2012;32(1):1-10. DOI: http://dx.doi.org/10.1016/j.iac.2011.10.001

13. Soares-Weiser K, Takwoingi Y, Panesar SS, Muraro A, Werfel T, Hoffmann-Sommergruber K, et al. The diagnosis of food allergy: a systematic review and meta-analysis. Allergy. 2014;69(1):76-86. DOI: http:// dx.doi.org/10.1111/all.12333

14. Boyce JA, Assa'ad A, Burks AW, Jones SM, Sampson HA, Wood RA, et al. Guidelines for the diagnosis and management of food allergy in the United States: Report of the NIAID-sponsored expert panel. J Allergy Clin Immunol. 2010;126(60):1-58. DOI: http://dx.doi.org/10.1016/j.jaci.2010.10.007

15. Eigenmann PA, Jae-Won O, Beyer K. Diagnostic testing in the evaluation of food allergy. Pediatr Clin N Am. 2011;58(2):351-362. DOI: http://dx.doi.org/10.1016/j.pcl.2011.02.003

16. Nolte H, Kowal K, DuBuske L. Overview of skin testing for allergic disease. Disponible en: http:// cursoenarm.net/UPTODATE/contents/mobipreview.htm?2/4/2113?source=HISTORY 
Camero-Martínez $\mathrm{H}$ et al. Reactividad cutánea hacia alérgenos alimentarios en pacientes alérgicos

17. Heinzerling L, Mari A, Bergmann KC, Bresciani M, Burbach G, Darsow U, et al. The skin prick testEuropean standards. Clin Transl Allergy. 2013;3(1):1-10. DOI: http://dx.doi.org/10.1186/2045-7022-3-3

18. Cianferoni A, Spergel JM. Food allergy; review, classification and diagnosis. Allergol Int. 2009;58(4):457466. DOI: http://dx.doi.org/10.2332/allergolint.09-RAI-0138

19. Medina-Hernández A, Huerta-Hernández RE, Góngora-Meléndez MA, Domínguez-Silva MG, MendozaHernández DA, Romero-Tapia SJ, et al. Perfil clínico-epidemiológico de pacientes con sospecha de alergia alimentaria en México. Estudio Mexipreval. Rev Alerg Mex. 2015;62(1):28-40. Disponible en: revistasmedicasmexicanas.com.mx/nieto/Alergia/2015/ene-mar/art.original_perfil.pdf

20. Sánchez J, Sánchez A. Epidemiology of food allergy in Latin America. Allergol Immunopathol (Madr). 2015;43(2):185-195. DOI: http://dx.doi.org/10.1016/j.aller.2013.07.001

21. Bedolla-Barajas M, Bedolla-Pulido TR, Camacho-Peña AS, González-García E, Morales-Romero J. Food hypersensitivity in Mexican adults at 18 to 50 years of age: A questionnaire survey. Allergy Asthma Immunol Res. 2014;6(6):511-516. DOI: http://dx.doi.org/10.4168/aair.2014.6.6.511

22. Maya-Epelstein J, Rosas-Vargas MA, Del-Río-Navarro B. Frecuencia de sensibilización a alimentos por pruebas cutáneas de prick-to-prick de parche en niños con enfermedades alérgicas. Rev Alerg Mex. 2012;59(3):123-130. 